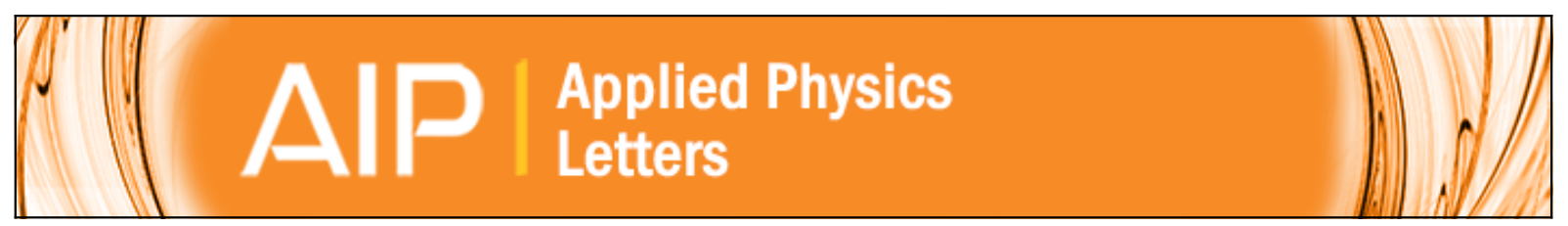

\title{
Effect of antimony on the deep-level traps in GalnNAsSb thin films
}

Muhammad Monirul Islam, Naoya Miyashita, Nazmul Ahsan, Takeaki Sakurai, Katsuhiro Akimoto, and Yoshitaka Okada

Citation: Applied Physics Letters 105, 112103 (2014); doi: 10.1063/1.4895940

View online: http://dx.doi.org/10.1063/1.4895940

View Table of Contents: http://scitation.aip.org/content/aip/journal/apl/105/11?ver=pdfcov

Published by the AIP Publishing

\section{Articles you may be interested in}

Identification of nitrogen- and host-related deep-level traps in n-type GaNAs and their evolution upon annealing J. Appl. Phys. 116, 013705 (2014); 10.1063/1.4886856

Defect study of molecular beam epitaxy grown undoped GalnNAsSb thin film using junction-capacitance spectroscopy

Appl. Phys. Lett. 102, 074104 (2013); 10.1063/1.4793430

Effect of carrier capture by deep levels on lateral photoconductivity of InGaAs/GaAs quantum dot structures J. Appl. Phys. 110, 043717 (2011); 10.1063/1.3626051

Effects of growth temperature on the structural and optical properties of $1.55 \mu \mathrm{m}$ GalnNAsSb quantum wells grown on GaAs

Appl. Phys. Lett. 87, 021908 (2005); 10.1063/1.1993772

Effects of thermal annealing on deep-level defects and minority-carrier electron diffusion length in Be-doped InGaAsN

J. Appl. Phys. 97, 073702 (2005); 10.1063/1.1871334

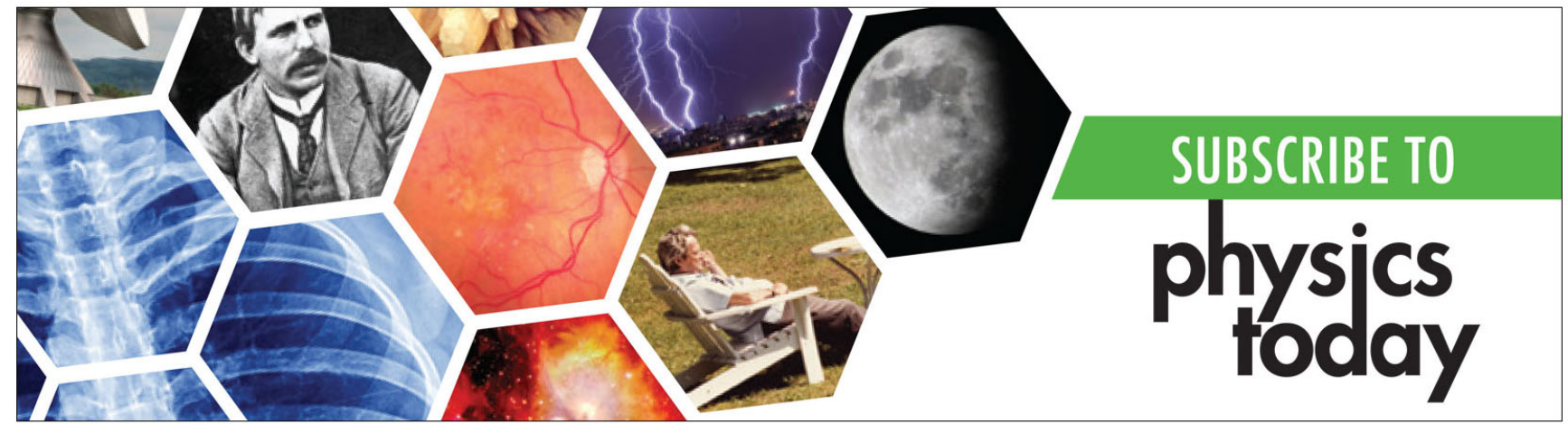




\title{
Effect of antimony on the deep-level traps in GalnNAsSb thin films
}

\author{
Muhammad Monirul Islam, ${ }^{1, a)}$ Naoya Miyashita, ${ }^{1}$ Nazmul Ahsan, ${ }^{1}$ Takeaki Sakurai, ${ }^{2}$ \\ Katsuhiro Akimoto, ${ }^{2}$ and Yoshitaka Okada $^{1}$ \\ ${ }^{1}$ Research Center for Advanced Science and Technology (RCAST), The University of Tokyo, 4-6-1 Komaba, \\ Meguro ku, Tokyo 153-8904, Japan \\ ${ }^{2}$ Institute of Applied Physics, University of Tsukuba, 1-1-1 Tennodai, Tsukuba, Ibaraki 305-8573, Japan
}

(Received 18 July 2014; accepted 5 September 2014; published online 16 September 2014)

\begin{abstract}
Admittance spectroscopy has been performed to investigate the effect of antimony $(\mathrm{Sb})$ on GaInNAs material in relation to the deep-level defects in this material. Two electron traps, $E 1$ and $E 2$ at an energy level 0.12 and $0.41 \mathrm{eV}$ below the conduction band $\left(E_{\mathrm{C}}\right)$, respectively, were found in undoped GaInNAs. Bias-voltage dependent admittance confirmed that $E 1$ is an interface-type defect being spatially localized at the GaInNAs/GaAs interface, while E2 is a bulk-type defect located around mid-gap of GaInNAs layer. Introduction of Sb improved the material quality which was evident from the reduction of both the interface and bulk-type defects. (C) 2014 AIP Publishing LLC. [http://dx.doi.org/10.1063/1.4895940]
\end{abstract}

GaInNAs has been considered as a promising material to realize third junction in the four-junction (4J) highefficiency tandem-solar cell due to its low band gap of $\sim 1 \mathrm{eV}$ and lattice matching to GaAs. ${ }^{1}$ However, crystal quality of this material deteriorates for higher concentration of nitrogen $(\mathrm{N})$ leading to the degradation of electrical and optical properties. ${ }^{2} \mathrm{~N}$-induced deep-level defects ${ }^{3,4}$ are usually considered for material degradation. Various studies reported that use of antimony $(\mathrm{Sb})$ in GaInNAs improves the material quality. $\mathrm{Sb}$ is usually considered to reduce point defects, promote two-dimensional growth, suppress phase-segregation via well-known surfactant effect, and it incorporates into the lattice in dilute limit forming GaInNAsSb. ${ }^{5,6}$ A recent study on intermediate band solar cell (IBSC) structure shows that incorporation of $\mathrm{Sb}$ into GaNAs improves the two-photon optical absorption. ${ }^{7}$ Nevertheless, knowledge about defects, their correlation with the GaInNAs growth conditions, in particular, with different $\mathrm{Sb}$-flux conditions, and their impact over the performance of GaInNAs-based devices have not yet reached up to the mark. Thus, a complete understanding of the defect-physics is indispensable for the successful application of this material. Various junction-capacitance techniques have been extensively used to characterize deeplevel defects in the semiconductors. ${ }^{8-10}$ In this letter, we performed admittance spectroscopy of molecular beam epitaxy (MBE)-grown GaInNAs thin films to investigate the effect of $\mathrm{Sb}$ on the deep-level traps in this material. Admittance spectroscopy ${ }^{11,12}$ provides thermal emission rates of electrically active defects in semiconductors from the variation of junction-capacitance and conductance as a function of temperature and frequency. These variation are due to the change in frequency of the measuring signal with respect to the time constant of the carrier trapping and de-trapping processes from the defect-level $\left(E_{\mathrm{t}}\right)$ around the point of the space-charge region where this level coincides with the

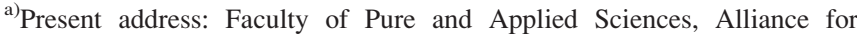
Research on North Africa (ARENA), University of Tsukuba, 1-1-1 Tennodai, Tsukuba, Ibaraki 305-8572, Japan. Electronic mail: islam.monir.ke@u.tsukuba.ac.jp.
}

Fermi level $\left(E_{\mathrm{F}}\right)$. The differential capacitance (or conductance) gives a peak at this crossing point at a temperature when the time constant associated with this capture and emission process matches the modulation frequency. The characteristic frequency, $\omega_{0}$, of this differential capacitance peak is related to the emission rate $\left(\tau_{0}{ }^{-1}\right)$ of trapped carriers according to the equation ${ }^{12}$

$$
\omega_{0}=2 \tau_{0}^{-1}=2 N_{\mathrm{C}, \mathrm{v}} v_{\mathrm{th}} \sigma_{\mathrm{t}} \exp \left(-E_{\mathrm{A}} / k T\right),
$$

where $N_{\mathrm{C}, \mathrm{V}} \propto T^{3 / 2}$ is the effective density of the band involved, $v_{\mathrm{th}} \propto T^{1 / 2}$ is the average thermal velocity, $\sigma_{\mathrm{t}}$ is the capture cross-section, and $E_{\mathrm{A}}$ denotes activation energy of the defect. Considering the temperature dependences of $N_{\mathrm{C}, \mathrm{V}}$ and $v_{\text {th }}$, Eq. (1) can be written as

$$
\omega_{\mathrm{O}}=2 \tau_{0}^{-1}=2 \xi_{0} T^{2} \exp \left(-E_{\mathrm{A}} / k T\right),
$$

where $\xi_{\mathrm{o}}$ is the temperature-independent pre-exponential factor.

$\mathrm{GaInNAs}(\mathrm{Sb})$ films in this study were grown by atomic hydrogen assisted MBE system. ${ }^{13}$ Dimeric $\mathrm{As}_{2}$ and monomeric $\mathrm{Sb}$ were supplied by valved cracker sources, while atomic $\mathrm{N}$ was supplied by a radio frequency (RF) nitrogen plasma cell. Three $p-i-n$ structures, consisting of $150 \mathrm{~nm} p$ $\mathrm{GaAs} / 300 \mathrm{~nm} i$-GaInNAs(Sb)/500 $\mathrm{nm} n$-GaAs, were subsequently grown on (001) $n^{+}$-GaAs substrate. A heavily doped $30 \mathrm{~nm} p^{+}$-GaAs cap layer was grown to facilitate ohmic contact. Ohmic contacts with $500 \mu \mathrm{m}$-diameters were made from AuZn. Undoped $i$-GaInNAs(Sb) layers were grown without $\mathrm{Sb}(\mathrm{Sb}=0)$, and also with $\mathrm{Sb}$ using two different fluxes $\left(\mathrm{Sb}=3 \times 10^{-8}\right.$ and $10 \times 10^{-8}$ Torr, measured as beam equivalent pressure) to investigate the effect of $\mathrm{Sb}$ on the film. Carrier concentration in the $p$-GaAs layer was set at $2 \times 10^{18} \mathrm{~cm}^{-3}$. Background dopant in the $i$-GaInNAs(Sb) layers was calculated using capacitance-voltage $(C-V)$ measurement. Doping level in the $i$-GaInNAs(Sb) layers with $\mathrm{Sb}$ flux $=0,3 \times 10^{-8}$, and $10 \times 10^{-8}$ Torr was estimated as $3.7 \times 10^{15}, 7.1 \times 10^{16}$, and $8.3 \times 10^{16} \mathrm{~cm}^{-3}$, respectively. Thus, depletion region can be considered to be extended into the $i$-GaInNAs( $\mathrm{Sb})$ layer in the structures, and we can safely 
assume that admittance spectroscopy probed the defect information from the $i$-GaInNAs( $\mathrm{Sb}$ ) layers in the structures. For defect characterization, samples were mounted inside a closed-cycle Janis cryostat. Admittance spectroscopy was carried out in the dark within the temperature range, 50-350 K using Agilent 4284A LCR meter. Amplitude of the $A C$ modulation voltage was kept as $25 \mathrm{mV}$ (rms), while modulation frequency was varied from $1 \mathrm{kHz}$ to $1 \mathrm{MHz}$. To study the bias dependence of the admittance spectroscopy, various $D C$ biases ranging from $0 \mathrm{~V}$ to $-2.0 \mathrm{~V}$ were applied during measurement.

Shown in Fig. 1(a) is the admittance spectra of all the $p-i-n$ structures. Measurement was performed at $D C$ bias, $V=0$. Each peak in the admittance spectra represents electrical response of a particular defect-level within the band-gap of the GaInNAs(Sb). Increase in the temperature shifts the admittance-peak (i.e., characteristic frequency, $\omega_{0}$ ) to the higher value. Thus, activation energy, $E_{\mathrm{A}}$, of a particular defect could be calculated from the slope of the corresponding Arrhenius plots, ${ }^{14} \ln \left(\omega_{\mathrm{o}} / T^{2}\right)$ versus 1/T (Figure 1(b)). Here, $T$ is the temperature of the admittance-peak at a particular frequency. Since admittance spectroscopy mainly determines the majority carrier trap centers, taking into account slightly $n$-type conductivity of the $i$-GaInNAs( $\mathrm{Sb})$ layers in this study, all the defect levels were assumed to be positioned below the $E_{\mathrm{C}}$ of the $i$-GaInNAs( $(\mathrm{Sb})$. Trap density was calculated using the principle as described by Walter et al. ${ }^{15}$
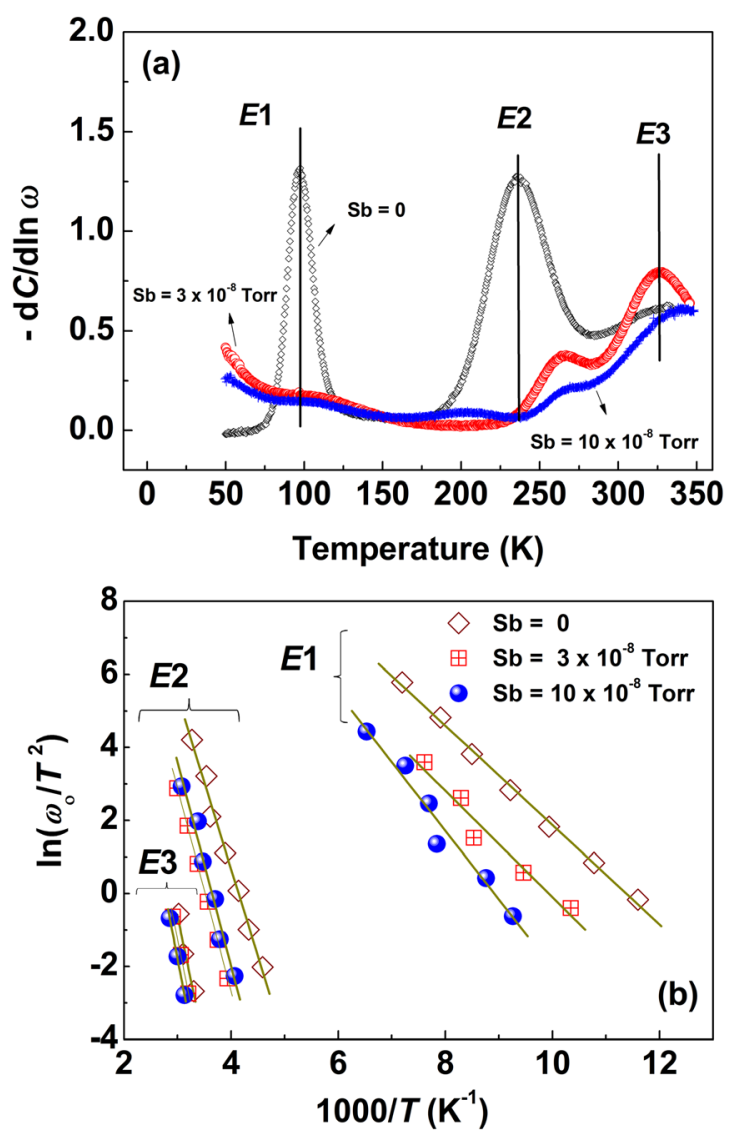

FIG. 1. (a) Admittance spectra of the GaInNAs(Sb)-based solar cell structures, where thermally active defects were determined in the $i$-GaInNAs(Sb) layer of the structure. (b) Arrhenius plots of the all the defect-peaks (E1,E2, and E3) measured from the temperature and frequency dependent admittance.
Capture cross-sections extrapolated to $T=\infty$ were calculated from slope of the Arrhenius plot using Eq. (1). During calculation of the capture cross-section, electron effectivemass ${ }^{16}$ was considered as $m_{\mathrm{e}}^{*}=0.09 m_{0}$, where $m_{0}$ is the free electron-mass.

In admittance spectra, GaInNAs film without $\mathrm{Sb}$ shows two dominant electron traps, $E 1$ and $E 2$ at an energy level, $E_{\mathrm{C}}-0.12$ and $E_{\mathrm{C}}-0.41 \mathrm{eV}$, respectively. Density of the $E 1$ and $E 2$ was estimated as $N_{\mathrm{t}} \sim 1.5 \times 10^{15}$ and $2.4 \times 10^{15} \mathrm{~cm}^{-3}$, respectively. Following the introduction of $\mathrm{Sb} \quad$ (flux $\sim 3 \times 10^{-8}$ Torr), $E 1$-defect almost vanished. Broader defect- $E 2$ was found to be reduced to at least one order of magnitude $\left(N_{\mathrm{t}} \sim 4.3 \times 10^{14} \mathrm{~cm}^{-3}\right)$, however, associated with a slightly higher activation energy of $0.48 \mathrm{eV}$ (i.e., right shifting of the admittance peak in the temperature scale). A defect, E3, was appeared around $330 \mathrm{~K}$ for all the samples having activation energy of $0.61-0.66 \mathrm{eV}$ below $E_{\mathrm{C}}$. However, requirement for the higher temperature (more than $350 \mathrm{~K})$ in the measurement restricted accurate determination of the E3-defect-parameters including defect activation energy. Therefore, we mainly focused on the $E 1$ and E2-defect in this study. For GaInNAsSb film, grown with higher Sb-flux $\left(10 \times 10^{-8}\right.$ Torr $)$, defect distribution was almost similar to that of lower $\mathrm{Sb}$-irradiated sample. Table I summarizes the defect parameters determined by the admittance spectroscopy of all the structures.

To understand the nature of the trap signatures found in the $i$-GaInNAs(Sb), we performed admittance spectroscopy of the GaInNAs (without $\mathrm{Sb}$ ) structure applying various $D C$ bias voltage. ${ }^{17,18}$ Shown in Fig. 2 is the reverse-bias dependence of the $E 1$-defect in the capacitance, $C$, as well as conductance, $G / \omega$ (here, $\omega=2 \pi f$ ), as a function of the modulating frequency, $f$, measured at $140 \mathrm{~K}$. $D C$ bias was varied from $0 \mathrm{~V}$ to $-2.0 \mathrm{~V}$ during measurement. For a particular applied bias, $C$ remains roughly constant (low-frequency value, $C_{\mathrm{lf}}$ ) up to the characteristic frequency, $\omega_{\mathrm{o}}$. Below $\omega_{\mathrm{o}}$, trapped charges at the intersection point of defect and $E_{\mathrm{F}}$ can follow the perturbation of the modulating signal with no loss of energy. However, as frequency becomes higher, response of the trapped charges at the intersection point may not be instantaneous, and there can be carriers above the $E_{\mathrm{F}}$ with energy higher than that of equilibrium. Consequently, phase lag appears in the $A C$ response of the charges with increasing

TABLE I. Summary of the thermally active defects in the $i$-GaInNAs(Sb) layers determined by the admittance spectroscopy. Experimental values of the thermal activation energy $\left(E_{\mathrm{A}}\right)$, thermal capture cross-section $\left(\sigma_{\mathrm{t}}\right)$, and trap concentration $\left(N_{\mathrm{t}}\right)$ of each defect are shown.

\begin{tabular}{lcccc}
\hline \hline Trap level & $\begin{array}{c}\text { Sb flux } \\
\left(\times 10^{-8} \text { Torr }\right)\end{array}$ & $\begin{array}{c}E_{\mathrm{A}}\left(\text { below } E_{\mathrm{C}}\right) \\
(\mathrm{eV})\end{array}$ & $\begin{array}{c}N_{\mathrm{t}} \\
\left(\mathrm{cm}^{-3}\right)\end{array}$ & $\begin{array}{c}\sigma_{\mathrm{t}} \\
\left(\mathrm{cm}^{2}\right)\end{array}$ \\
\hline E1 & 0 & 0.12 & $1.5 \times 10^{15}$ & $1.2 \times 10^{-14}$ \\
& 3 & 0.16 & $\ldots$ & $3.2 \times 10^{-14}$ \\
E2 & 10 & 0.13 & $\ldots$ & $3.0 \times 10^{-15}$ \\
& 0 & 0.41 & $2.4 \times 10^{15}$ & $5.8 \times 10^{-13}$ \\
& 3 & 0.48 & $4.3 \times 10^{14}$ & $6.2 \times 10^{-13}$ \\
E3 & 10 & 0.48 & $4.5 \times 10^{14}$ & $5.0 \times 10^{-13}$ \\
& 0 & 0.61 & $8.2 \times 10^{14}$ & $1.4 \times 10^{-12}$ \\
& 3 & 0.62 & $1.0 \times 10^{15}$ & $1.2 \times 10^{-12}$ \\
\hline \hline
\end{tabular}




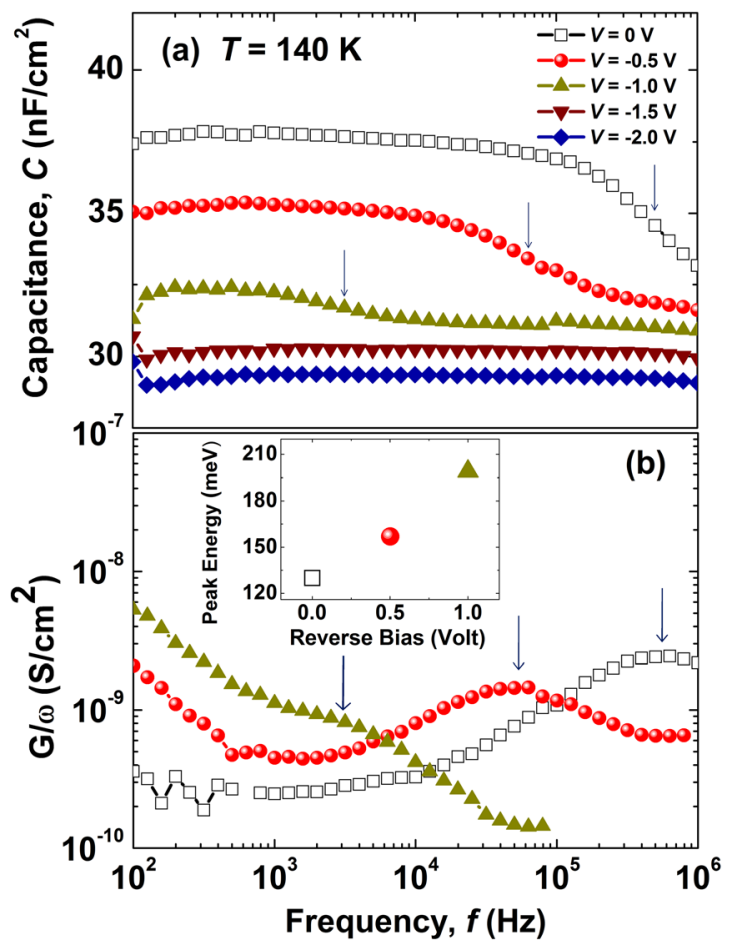

FIG. 2. (a) $D C$ bias dependence of the $E 1$-peak in the capacitance spectra as a function of the modulating frequency, $f(\mathrm{~b}) D C$ bias dependence of the $E 1$ peak in the normalized conductance $G / \omega$ spectra as a function of the modulating frequency, $f$. Measurement was done at $140 \mathrm{~K}$. Applied $D C$ bias was varied from $0 \mathrm{~V}$ to $-2.0 \mathrm{~V}$ during the measurement. Shifting of peak at $G / \omega$ $f$ plot with applied reverse bias has been indicated with an arrow. Inset of Fig. 2 (b) shows shifting of the peak- $E 1$ with the applied bias in energyscale.

frequency. It generates real part in the admittance i.e., conductance term $(G)$ associated with this loss. The capacitance shows a step and decreases to a high-frequency value, $C_{\mathrm{hf}}$. At the capacitance-step, normalized conductance $(G / \omega)$ shows a peak having a maximum at the frequency, $\omega_{\mathrm{o}}$, when this loss is maximum. The peak at $G / \omega$ is related to the capacitance-step according to the linear response theory ${ }^{10}$ and is equal to $1 / 2\left(C_{\mathrm{lf}}-C_{\mathrm{hf}}\right)$. It should be noted that at sufficiently high reverse bias, the step in the capacitance in Fig. 2(b) disappears. Decrease in the $C$ at all the frequency region with applying reverse bias can be attributed to the increase in the depletion width. In case of $G / \omega-f$ curve (Fig. 2(b)), with applying reverse bias, a shift in the peak position to the lower frequency was observed. We can explain the peak shift as follows.

Previously, we studied band-diagram model for the identification of defect-types in GaInNAs material. ${ }^{17}$ With applying a $D C$ reverse-bias, there will be an increase in separation between the band-edge ( $E_{\mathrm{C}}$ in this case) and the $E_{\mathrm{F}}$. If there exist any interface states (i.e., defects which are energetically continuous throughout the band-gap) at the heterojunction, there will also be a corresponding increase in the separation between $E_{\mathrm{C}}$ and crossing point of these interface states with $E_{\mathrm{F}}$. Therefore, energy depth, $E_{\mathrm{A}}$ (i.e., the activation energy), of those states will be increased with reverse bias. This will result in the decrease of the emission rate $\left(e_{\mathrm{T}}=\tau_{0}{ }^{-1}\right)$ of the trapped carriers from these interface states. Since $\omega_{\mathrm{O}}$ is related to the $e_{\mathrm{T}}$ according to the Eq. (1), decreased $e_{\mathrm{T}}$ of the trapped charges will make a lower shift in the $\omega_{\mathrm{o}}$ (i.e., peak of $G / \omega-f$ curve) with reverse bias. In contrast, in case of bulk-type defects, defect-levels are spatially continuous along the depth of the film. Therefore, energetic distance between the crossing point (i.e., intersection between defect-level and $E_{\mathrm{F}}$ ) and the $E_{\mathrm{C}}$ remains same irrespective of the applied bias. Consequently, there should be no such shifting of the admittance-peak with change in the bias voltage.

Therefore, considering the above principle, left shifting of the peak- $E 1$ at $G / \omega-f$ curve in Fig. 2(b) can be attributed to the decreased $e_{\mathrm{T}}$ due to applied reverse bias, i.e. $E 1$-defect can be considered as the interface-type defect. In addition, inset of Fig. 2(b) shows shifting of the peak-E1 with the applied bias in energy scale. Frequency-scale has been changed to energy-scale according to Eq. (2) where preexponential factor, $\xi_{0}$, of the peak- $E 1$ was calculated from the Arrhenius plot of the corresponding peak. A significant increase of the energy-separation between E1-states and band edge with applied reverse-bias has been observed. This phenomenon rules out any probability of potential fluctuation around the determination point of defect position which may lead to the change in energy-depth up to several meV. Furthermore, $e_{\mathrm{T}}$ of the trapped carriers from the defect levels can be enhanced by the strong electric field which is usually explained with the Pool-Frenkel effect, phonon assisted tunneling, and the direct tunneling. ${ }^{19,20}$ In all these cases, energy-depth of the defects will be lowered with applied reverse bias (i.e., with an increase in electric field) which is in contrast to the observed increase of the energy-depth in this study. Moreover, unlike deep level transient spectroscopy (DLTS) ${ }^{8}$ where emission occurs over the whole depletion region of the junction, carrier emission in the admittance spectroscopy occurs only around the depth of the crossover, where the $E_{\mathrm{F}}$ crosses the defect-level. Therefore, effect of the electric field on the emission process, if there is any, should be negligible and we can ignore this effect in our study.

Shown in Fig. 3 is the Arrhenius plot of the emission rates $\left(\omega_{0} \sim e_{\mathrm{T}}\right)$ of the defect-E1 measured at various bias voltage. For a particular bias condition, Arrhenius plot was calculated from the peak of the $G / \omega-f$ curve measured at various temperature and frequency. It is evident from the figure that for a particular temperature, $e_{\mathrm{T}}$ decreases (i.e., energydepth of defect increases) with an increase in reverse bias.

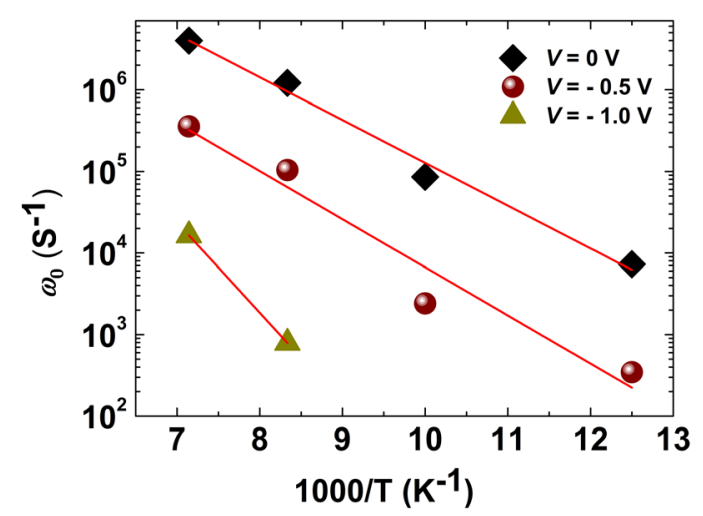

FIG. 3. Emission rates $\left(\omega_{0} \sim e_{\mathrm{T}}\right)$ of the defect- $E 1$ measured at various bias voltage and temperature. 


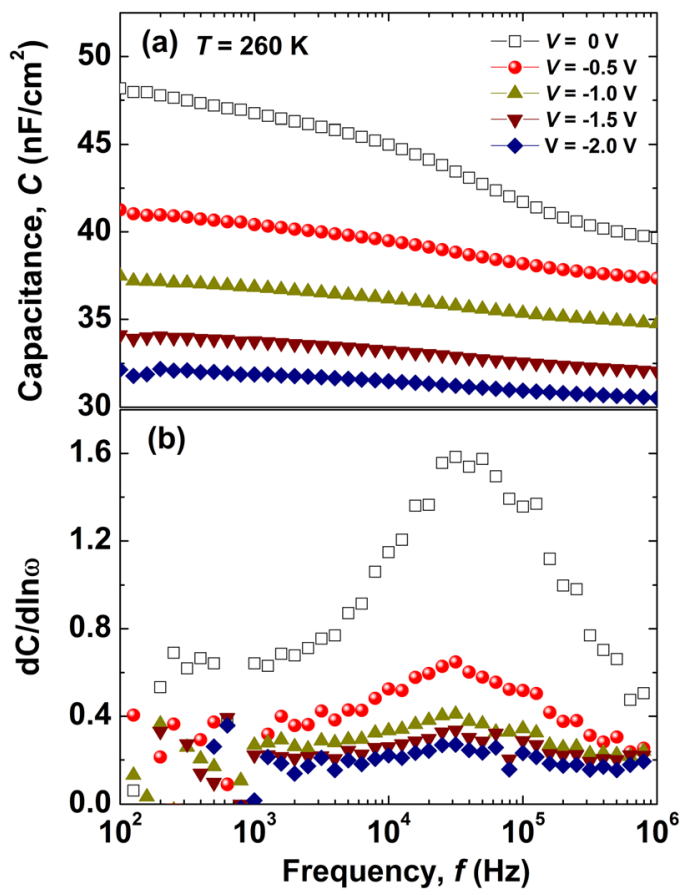

FIG. 4. (a) $D C$ bias dependence of the E2-peak in the capacitance spectra measured as a function of the modulating frequency, $f$. (b) $D C$ bias dependence of the E2-peak in the differential capacitance, $\mathrm{d} C / \mathrm{d} \ln \omega$ spectra measured as a function of the modulating frequency, $f$. Measurement was done at $260 \mathrm{~K}$. Applied $D C$ bias was varied from $0 \mathrm{~V}$ to $-2.0 \mathrm{~V}$.

All the above experimental evidences strongly suggest that the $E 1$ is an interface-type defect at the GaAs/GaInNAs(Sb) hetero-interface, i.e., $E 1$ is spatially localized, while energetically continuous throughout the band-gap of the $i$-GaInNAs(Sb). It is to be mentioned that since depletion region is extended in to the $i$-GaInNAs( $(\mathrm{Sb})$ from the junction between top $p$-GaAs and $i$-GaInNAs( $\mathrm{Sb})$, primarily, we can assume that $E 1$ traps are localized at the top $p$-GaAs/ $\mathrm{GaInNAs}(\mathrm{Sb})$ hetero-interface.

Later, we investigated defect-type of E2-peak in the $i$ GaInNAs (without $\mathrm{Sb}$ ). Figure 4 shows bias dependent $C$ and $\mathrm{d} C / \mathrm{d} \ln \omega$ as a function of frequency as measured in $T=260 \mathrm{~K}$. Due to large contribution of $D C$ part in the conductance-value, peaks in the $G / \omega-f$ plot were difficult to be distinguished. Therefore, we have selected differential capacitance value (i.e., $\mathrm{d} C / \mathrm{d} \ln \omega$ ) to determine the defect-type for $E 2$. Unlike defect- $E 1$, no such shifting of the peak at $\mathrm{d} C / \mathrm{d} \ln \omega-f$ plot with applying $D C$ bias was observed. Considering the principles as discussed above, it suggests that $E 2$ is a bulk-type defect i.e., E2 is spatially homogeneous along the depth of the material with single energy level.

From Table I, it is clear that with introduction of the Sb in GaInNAs material, density of both the interface and bulktype defects was significantly reduced. Moreover, it is to be noted that E2-defect is located around mid-gap (0.40-0.44 of $\mathrm{E}_{\mathrm{g}}$ ) of the $i$-GaInNAs $(\mathrm{Sb})$ layer and is associated with a large capture cross-section of $\sim 10^{-13} \mathrm{~cm}^{2}$. Therefore, it is highly plausible that E2-defect acts as an efficient recombination center in the solar cell. Thus, reduction of both $E 1$ and E2defects indicates improved material properties of Sbirradiated GaInNAs.
As reported, improvement of the material-quality of bulk-GaInNAs with introduction of optimum amount of $\mathrm{Sb}$ has been manifested in the improved photoluminescence (PL)-intensity, as well as in reduced peak-energy shift (i.e., reduced carrier localization by $\mathrm{N}$-related sates) in the temperature dependent PL measurement. ${ }^{13,21}$ On the other hand, improved interface-properties after Sbirradiation have been confirmed by the formation of atomically flat interface between GaInNAs/GaAs single or multiple quantum well. ${ }^{22,23}$ In addition, reflection highenergy electron diffraction (RHEED) and atomic force microscope (AFM) have been routinely used to confirm better surface reconstruction and surface morphology in the Sb-mediated GaInNAs films. ${ }^{21,23}$ Better surface morphology might be resulted from the improved interfaceproperties at the GaInNAs(Sb)/GaAs hetero interface. All these experimental evidences support the improvement of interface as well as bulk properties of the GaInNAs samples with incorporation of $\mathrm{Sb}$. Although further study is required for more confirmation, improvement of the GaInNAs(Sb) quality as studied by various authors can partly be attributed to the reduction of the $E 1$ and E2defect, studied in this letter.

In summary, admittance spectroscopy of the GaInNAs(Sb)-based structures shows two majority electron traps, $E 1$ and $E 2$ at an energy level $E_{\mathrm{C}}-0.12$ and $E_{\mathrm{C}}-$ $0.41 \mathrm{eV}$, respectively, in the $i$-GaInNAs(Sb) layer. Biasvoltage dependent admittance measurement showed that $E 1$ is an interface-type defect, while $E 2$ is a bulk-type defect. A significant reduction of these defects was observed when GaInNAs film were irradiated with $\mathrm{Sb}$ flux. Improvement of the GaInNAs film- quality following the introduction of $\mathrm{Sb}$ as studied by many authors in the literature can roughly be attributed to the reduction of both the interface $(E 1)$ and the bulk-type defect $(E 2)$ as found in our study.

This work was performed under Research and Development on Innovative Solar Cells (SOLAR QUEST) program supported by the New Energy and Industrial Technology Development organization (NEDO) and the Ministry of Economy, Trade and Industry (METI), Japan.

${ }^{1}$ M. Kondow, T. Kitatani, M. C. Larson, K. Nakahara, K. Uomi, and H. Inoue, J. Cryst. Growth 188, 255 (1998).

${ }^{2}$ D. J. Friedman, J. F. Geisz, S. R. Kurtz, and J. M. Olson, J. Cryst. Growth 195, 409 (1998)

${ }^{3}$ S. R. Kurtz, A. A. Allerman, C. H. Seager, R. M. Sieg, and E. D. Jones, Appl. Phys. Lett. 77, 400 (2000).

${ }^{4}$ S. G. Spruytte, C. W. Coldren, J. S. Harris, W. Wampler, P. Krispin, K. Ploog, and M. C. Larson, J. Appl. Phys. 89, 4401 (2001).

${ }^{5}$ H. Shimizu, K. Kumada, S. Uchiyama, and A. Kasukawa, Electron. Lett. 36, 1379 (2000)

${ }^{6}$ J. C. Harmand, L. H. Li, G. Patriarche, and L. Travers, Appl. Phys. Lett. 84, 3981 (2004).

${ }^{7}$ N. Ahsan, N. Miyashita, M. Monirul Islam, K. Man Yu, W. Walukiewicz, and Y. Okada, IEEE J. Photovoltaics 3, 730 (2013).

${ }^{8}$ D. V. Lang, J. Appl. Phys. 45, 3023 (1974).

${ }^{9}$ M. Monirul Islam, N. Miyashita, N. Ahsan, T. Sakurai, K. Akimoto, and Y. Okada, J. Cryst. Growth 378, 57 (2013).

${ }^{10}$ M. Monirul Islam, N. Miyashita, N. Ahsan, and Y. Okada, Appl. Phys. Lett. 102, 074104 (2013).

${ }^{11}$ G. I. Roberts and C. R. Crowell, J. Appl. Phys. 41, 1767 (1970).

${ }^{12}$ D. L. Losee, J. Appl. Phys. 46, 2204 (1975). 
${ }^{13}$ N. Miyashita, S. Ichikawa, and Y. Okada, J. Cryst. Growth 311, 3249 (2009).

${ }^{14}$ J. L. Pautrat, B. Katircioglu, N. Magnea, D. Bensahel, J. C. Pfister, and L. Revoil, Solid-State Electron. 23, 1159 (1980).

${ }^{15}$ T. Walter, R. Herberholz, C. Müller, and H. W. Schock, J. Appl. Phys. 80, 4411 (1996).

${ }^{16}$ Z. Pan, L. H. Li, Y. W. Lin, B. Q. Sun, D. S. Jiang, and W. K. Ge, Appl. Phys. Lett. 78, 2217 (2001).

${ }^{17}$ M. Monirul Islam, N. Miyashita, N. Ahsan, T. Sakurai, K. Akimoto, and Y. Okada, J. Appl. Phys. 112, 114910 (2012).
${ }^{18}$ M. Sakhaf and M. Schmeits, J. Appl. Phys. 80, 6839 (1996).

${ }^{19}$ J. Frenkel, Phys. Rev. 54, 647 (1938).

${ }^{20}$ R. M. Hill, Philos. Mag. 23, 59 (1971).

${ }^{21}$ N. Miyashita, N. Ahsan, and Y. Okada, Sol. Energy Mater. Sol. Cells 111, 127 (2013).

${ }^{22}$ D. Wu, Z. Niu, S. Zhang, H. Ni, Z. He, Z. Sun, Q. Han, and R. Wu, J. Cryst. Growth 290, 494 (2006).

${ }^{23}$ X. Yang, J. B. Héroux, L. F. Mei, and W. I. Wang, Appl. Phys. Lett. 78, 4068 (2001). 